\title{
The Learning Curve of the Beginner Surgeon with Supervisor for Laparoscopic Totally Extraperitoneal Repair
}

\author{
Jaeho Lee, M.D., Myung-Jin Kim, M.D., Kyung-Yul Hur, M.D., Ph.D. \\ Department of Surgery, College of Medicine, Soonchunhyang University, Seoul, Korea
}

\begin{abstract}
Purpose: Despite acceptance of laparoscopic totally extraperitoneal (TEP) repair for repair of inguinal hernia, it is still considered to require a prolonged learning curve. We hypothesized that there would be differences in the learning curve for laparoscopic TEP repair among beginner surgeons with or without a supervisor. The current study was conducted to observe the learning curve for laparoscopic TEP repair performed by a surgeon without a supervisor.
\end{abstract}

Methods: A retrospective analysis of the consecutive 143 patients was conducted to document patients' demographics, hernia types, operative time, and operative outcomes after the operation. Patients were divided into 7 consecutive groups, each comprising 20 patients.

Results: The mean age of the study population was 52.5 years $( \pm 18.9 \mathrm{SD}$ ), ranging from 16 to 89 years. The learning curve of this technique was almost horizontal throughout the time course. The mean operative time was quite stable among each group. Comparison of the mean operation time and the length of hospital stay among each group did not show significant differences. There was one recurrence in group $\mathrm{V}$.

Conclusion: The learning curve for laparoscopic TEP repair is not that prolonged if there is an experienced specialized supervisor and opportunities to observe the entire procedure performed by the supervisor are available. It is not the laparoscopic instrument skills but the way the procedure is performed that is important for successful outcomes.

Keywords: Laparoscopic TEP repair, Inguinal hernia, Learning curve

This is an Open Access article distributed under the terms of the Creative Commons Attribution Non-Commercial License (http:// creativecommons.org/licenses/by-nc/4.0/) which permits unrestricted non-commercial use, distribution, and reproduction in any medium, provided the original work is properly cited.
Received August 31, 2015

Revised October 28, 2015

Accepted November 2, 2015

Corresponding author

Kyung-Yul Hur

Department of Surgery,

Soonchunhyang University Hospital, 59, Daesagwan-ro, Yongsan-gu,

Seoul 04401, Korea

Tel: +82-2-709-9114

Fax: +82-2-749-0449

E-mail: hurusa@hanmail.net

\section{INTRODUCTION}

Laparoscopic totally extraperitoneal (TEP) repair has gained its acceptance to repair inguinal hernia as it is safe and effective in treating inguinal hernias with the advantages of less pain, better cosmesis, and a more rapid recovery. ${ }^{1-3}$ However, it has still been considered difficult to learn, that is, it is considered to need prolonged learning curve. ${ }^{4}$ Nevertheless, many hernia surgeons have practiced it, standardized its steps, and established the procedure.
A learning curve has been defined as the number of operations required for reaching a plateau in operating time and complication rate. ${ }^{5}$ The learning curve for laparoscopic TEP repair has regarded quite longer or prolonged compared with other laparoscopic surgeries because of indirect visualization of unfamiliar anatomy-inside out-through laparoscope. ${ }^{6}$ Several studies have reported that the learning curve for laparoscopic TEP repair was about 50 to $80^{7-9}$

We hypothesized that there would be differences in the learning curve for laparoscopic TEP repair between beginner 
surgeons with or without supervisor and their pupils. The present study was undertaken to observe the learning curve for laparoscopic TEP repair performed by the surgeon without supervisor.

\section{MATERIALS AND METHODS}

As an ordinary resident training course with intra-department rotating system, there had been three period of attending to hernia department for 3 month a year during the total of four-year-training course. Thereafter, one of the authors has performed laparoscopic TEP repair for inguinal hernia in 227 patients since 2011. The assistant who took charge as a scopist was a surgical resident or physician's assistant (P. A.). The first case was performed after assisting 50 cases of laparoscopic TEP repairs performed by experienced surgeon during the training course as a surgical resident and the all case was performed by single surgeon without supervisor. Among them, we excluded 84 patients followed up less than one year because most recurrences after hernia surgery occur within one year. A retrospective analysis of the consecutive 143 patients was performed. All the medical records and personal brief reports by the surgeon were reviewed to document patients' demographics, hernia types, operative time, and perioperative outcomes after the operation.

\section{Laparoscopic TEP repair}

We have previously described our standardized procedure of laparoscopic TEP repair. ${ }^{10}$ Briefly describing, a laparoscope is introduced beneath the rectus abdominis muscle through the incision at the inferior edge of the umbilicus. A space between the rectus muscle and posterior rectus sheath was created by blunt dissection with Endo-Peanut (US Surgical, Norwalk, CT, USA) and a 45 degree laparoscope. After medial dissection of widening the space of Retzius, we identified external iliac vessels and inferior epigastric vessels. Then, we isolated the spermatic cord with two Endo-Peanuts. The hernia sac was gradually dissected and freed from the spermatic cord in cases of indirect hernias. A direct hernia sac, if present, was reduced easily by simple traction, and then fixed to the Cooper's ligament or pubic bone with a fixing device to prevent seroma collection or slippage of the mesh. Dissection was continued by gentle traction on the cord elements to parietalize the cord structures. The hernia sac or proximal portion of the sac if the sac was cut in the middle had to be peritonealized, which means that it is pushed back into the abdominal cavity as far as possible to procure enough preperitoneal space. After performing the lateral dissection sufficiently, enough size of mesh to cover entire myopectineal orifice of Fruchaud was introduced through the $10 \mathrm{~mm}$ trocar and fully laid out to reinforce the entire myopectineal orifice. We fixed the prosthetics on pubic bone or rectus muscle using helical metal tackers (Protac, Tyco Healthcare, Norwalk, CT). Desufflation was always performed with care under constant vision to ensure of not being folded of the mesh.

\section{Postoperative assessment}

Patients were resumed tolerable diet and light activities on the day of operation after being fully awake from anesthetic. All patients were prescribed oral pain killer 3 times a day. A length of hospital stay refers to the total number of nights spent in hospital after the operation. Patients were followed up at the out-patient department 7 to 10 days after the operation. They were provided the phone number of the hernia center of our institute in case they have any question about the surgery such as worsening pain, swelling on groin area, etc.

\section{Statistical analysis}

Statistical analysis was performed through the computer software (IBM SPSS/PC ver. 19.0; SPSS, Chicago, IL, USA). Continuous values were expressed as mean \pm standard deviation (SD). Comparison of data among the seven groups was performed by independent Student t-test and chi-square test where appropriate. A $p$ value of $<0.05$ was regarded as significant.

\section{RESULTS}

\section{Patient demographics and characteristics of inguinal hernia}

The mean age of the study population was 52.5 years $( \pm 18.9$ $\mathrm{SD})$, with a range from 16 to 89 years. There were 134 men and 9 women. There were 99 unilateral hernias and 44 bilateral hernias. There were 56 cases of right inguinal hernia and 43 cases of left inguinal hernia in the unilateral hernia group. The types of hernia were as follows: 83 indirect, 47 pantaloon, 57 direct. In addition, there was one femoral hernia, 9 recurrent hernias, and 2 cases of metachronous inguinal hernia. The types of hernia were expressed only in index hernia. Three patients had enlarged femoral ring. The mean follow up period was 27 months (range, 12 48).

Patients were divided into 7 consecutive groups simply in the time order of surgery, each comprising 20 patients in group I VI, and 23 patients in group VII. Table 1 shows the demographics of the patients and characteristics of inguinal hernias for each group. 
Table 1. Comparison of demographics, and characteristics of hernia among the seven groups of patients

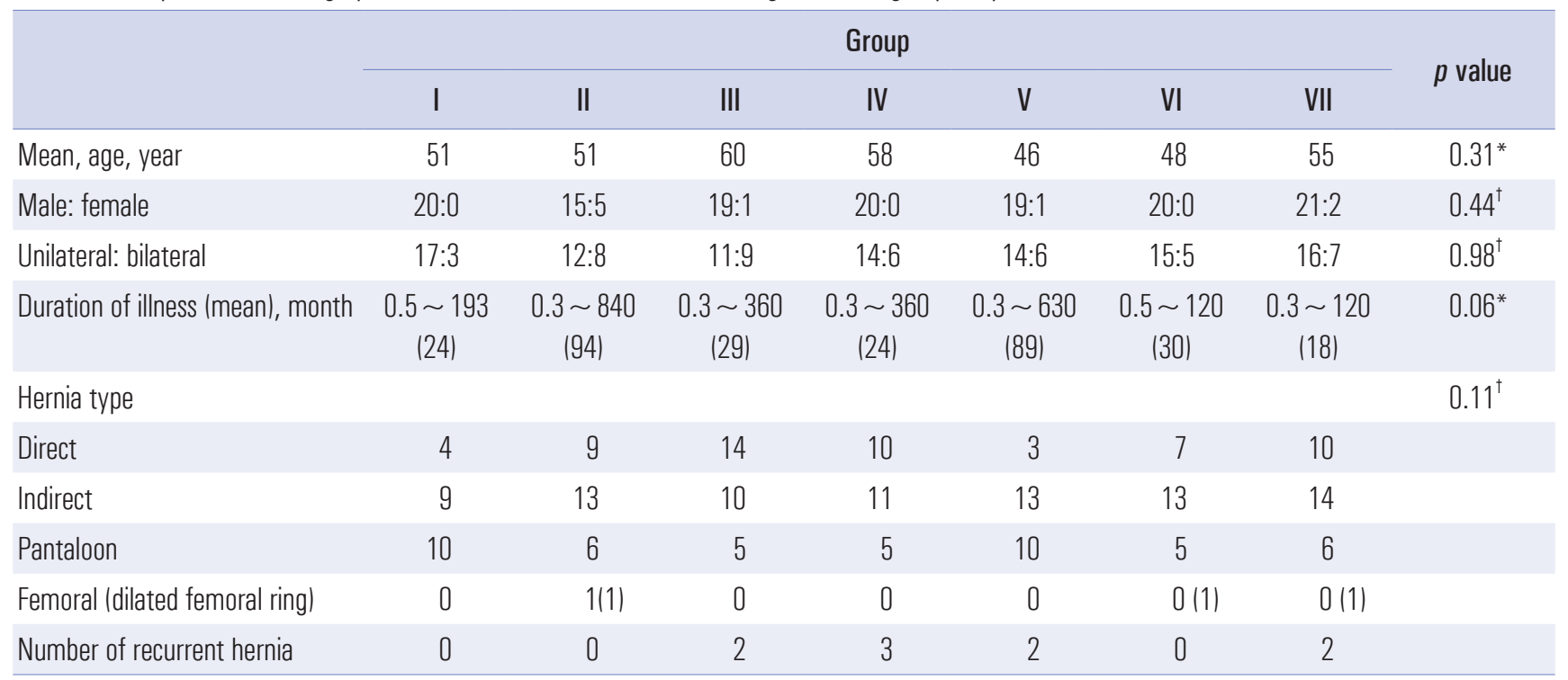

${ }^{*}$ Student's t test, ${ }^{\dagger}$ Chi-square test.

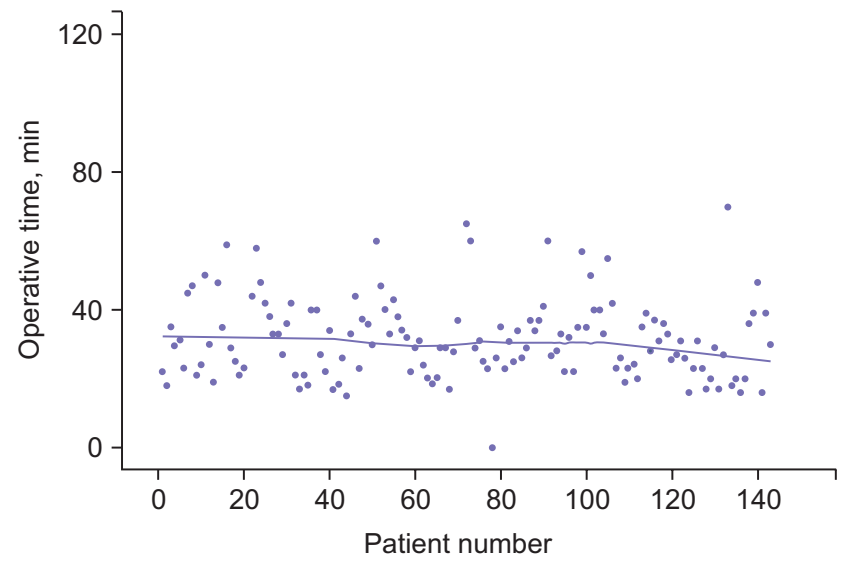

Fig. 1. A scatter plot of the operative time and the learning curve for laparoscopic TEP repair.

\section{Comparison of perioperative outcomes}

Fig. 1 shows the scatter plot of the operative time for the laparoscopic TEP repair. The learning curve of this technique in the present study is shown on the plot. It was almost horizontal through the time course.

There is no significant differences between the mean duration of surgery among each group $(p=0.21)$. The mean duration of surgery was quite stable among each group. It took about 30 minutes to perform laparoscopic TEP repair.

The overall mean length of hospital stay was $1.1 \pm 0.6$ days (range, $0 \sim 7$ ). Almost all patients discharged on the first day after the operation ( $n=132,92.3 \%)$. Comparison of this outcome among each groups did not show significant differences, either $(p=0.18)$.

Although 10 patients had history of previous abdominal surgery or obese, there was no conversion to other procedure such as transabdominal preperitoneal (TAPP) or open anterior approach. There were no visceral or major vascular injuries.

The intraoperative complication rate was $3.5 \% \quad(n=5)$, which required assistance by experienced senior surgeon (Hur KY). Three cases in the group I and II needed to switch the operator to the supervisor. All of those were minor and resolved through laparoscopic technique. There was no patient who complained of chronic pain. The mean length of followup was 27 months, ranged from 12 months to 48 months. There was one recurrence ( $93^{\text {rd }}$ case).

Table 2 compares the perioperative outcomes among the seven groups of patients.

\section{DISCUSSION}

Laparoscopic TEP repair for inguinal hernia has gained worldwide acceptance and has become, in many centers, the first choice for inguinal hernia repair. ${ }^{10}$ The advantages of this approach are as follows: reduced postoperative pain and earlier return to usual activities. In addition, one of the biggest merits of this procedure is its ability to show entire myopectineal orifice, so it is possible to repair any unexpected hernias simultaneously. ${ }^{10-12}$ Moreover, anteriorly approaching open procedure such as bilayer hernia repair with Prolene 
Table 2. Comparison of perioperative outcomes among the seven groups of patients

\begin{tabular}{|c|c|c|c|c|c|c|c|c|}
\hline & & & & Group & & & & \\
\hline & I & ॥ & III & IV & V & VI & VII & $\rho$ value \\
\hline $\begin{array}{l}\text { Mean, operative time, min } \\
(\min \sim \max )\end{array}$ & $\begin{array}{c}31.8 \\
(18 \sim 59)\end{array}$ & $\begin{array}{c}33.7 \\
(17 \sim 58)\end{array}$ & $\begin{array}{c}32.9 \\
(17 \sim 60)\end{array}$ & $\begin{array}{c}28.9 \\
(17 \sim 65)\end{array}$ & $\begin{array}{c}33.4 \\
(22 \sim 60)\end{array}$ & $\begin{array}{c}33.0 \\
(19 \sim 55)\end{array}$ & $\begin{array}{c}27.8 \\
(16 \sim 70)\end{array}$ & $0.21^{*}$ \\
\hline $\begin{array}{l}\text { Mean hospital stay, days } \\
(\min \sim \max )\end{array}$ & $\begin{array}{c}1.3 \\
(1 \sim 7)\end{array}$ & $\begin{array}{c}1.3 \\
(1 \sim 4)\end{array}$ & $\begin{array}{c}1.0 \\
(1 \sim 2)\end{array}$ & $\begin{array}{c}1.1 \\
(1 \sim 3)\end{array}$ & $\begin{array}{c}1.2 \\
(1 \sim 3)\end{array}$ & $\begin{array}{c}1.0 \\
(0 \sim 1)\end{array}$ & $\begin{array}{c}1.0 \\
(0 \sim 1)\end{array}$ & $0.18^{*}$ \\
\hline Intraoperative complications & 2 & 1 & 0 & 1 & 1 & 0 & 0 & $0.10^{\dagger}$ \\
\hline Conversion & 0 & 0 & 0 & 0 & 0 & 0 & 0 & \\
\hline Recurrence & 0 & 0 & 0 & 0 & 1 & 0 & 0 & $0.65^{\dagger}$ \\
\hline
\end{tabular}

Hernia System (PHS) would become easier after getting used to this procedure - laparoscopic TEP repair - because posterior aspect of inguinal anatomy is able to be pictured more detailed.

On the other hand, the main debates against laparoscopic inguinal hernia repair are as follows: requiring general anesthesia, and more importantly, difficult in learning the procedure. ${ }^{13-15}$ It has been considered to have prolonged learning curve.

The concept of the learning curve was first introduced in the manufacturing industries. ${ }^{16}$ It is discovered that unit costs decrease exponentially as a result of cumulative output. When data of cost was plotted on a scatter plot, the line of best fit took the form of a power function. This line was called the 'learning curve.' It has also been loosely used to describe the time course required to acquire the stabilization of operating time and complication rates in the medical science, especially in the surgical field. ${ }^{5,16}$

There have been numerous reports on the learning curve for laparoscopic procedure in the surgical field in the era of minimally invasive surgery since the early 1990 s. $^{17-19}$ It is usually required dozens of cases to stabilize the operating time and postoperative outcomes: about 20 to 30 cases for laparoscopic cholecystectomy, 30 to 70 cases for laparoscopic colorectal surgery, and approximately 50 cases for laparoscopy assisted distal gastrectomy.

There have also been several studies on the learning curve for laparoscopic TEP repair ${ }^{7-9}$; one concluded a learning curve of 80 cases,' another of $20-50$ cases, ${ }^{8}$ and the other of 60 cases. ${ }^{9}$

Although there is no great difference numerically in the learning curve for the laparoscopic procedures in the surgical field including laparoscopic TEP repair, laparoscopic TEP repair has considered more difficult to learn because of its unfamiliar access route, so-called 'inside out,' and narrow working space., ${ }^{920}$

It is not surprising that there is difference in achieving competence in surgical skill in cases of having experienced supervisor or not. A beginner with experienced supervisor could draw shorter learning curve. However, almost all learning curves take the form of a power function even if an access route is familiar and there is an experienced supervisor. Interestingly, however, our results showed different shape of the learning curve as using the operating time as a parameter. The curve was almost linear, not the form of a power functions, from the beginning. The author observed 50 cases of laparoscopic TEP repair by assisting the supervisor as a chief resident and a fellow. The supervisor had commented on the key steps of the procedure and on the anatomic landmark important to the procedure during the period.

The first case was performed for 34-year-old male with overweight $(\mathrm{BMI} \fallingdotseq 25)$. In general, a procedure for younger or obese patient is considered more difficult because there would be lots of fat tissue and denser connective tissue in the preperitoneal space. However there was no great difficulty in identifying the inferior epigastric vessels, the spermatic cord, and the indirect hernia sac. It may be due to being familiar with the preperitoneal anatomy after observing 50 cases.

The first case which needed assistance by the supervisor was the $10^{\text {th }}$. The reason assistance was needed was a defect of the peritoneum and loss of working space in the early phase of the procedure. The only thing the supervisor did was sticking the Veress needle at the side and connecting suction canula to it. The total number of the cases which needed intraoperative assistance by the supervisor or needed to switch the opearator to the supervisor were three. Both were in the first two groups. Other intraoperative complications in Group IV and V were minor bleeding that was controlled easily.

The supervisor is not necessarily to be involved in the 
operation as a scopist or an assistant. She or he can give the beginner hint about the key steps of the procedure if needed, so-called as 'on-site mentoring.'

Haidenberg et al analyzed outcomes of laparoscopic TEP repair performed by surgical trainees and reported three percent of recurrence rate, and $75 \%$ of the recurrence occurred within the first 40 cases. ${ }^{8}$ We have experienced only one recurrence (the $93^{\text {rd }}$ case in Group V), which occurred within one week after the surgery $(0.7 \%)$. Laparoscopic TEP repair was successfully performed again for this recurrent case. The mechanism of recurrence was sliding of the indirect sac behind the mesh after deflation of the extraperitoneal space.

The important thing is not the laparoscopic instrument skills. Today's young surgeons are very skillful in dealing with laparoscopic instruments and in performing laparoscopic techniques because many specialist practitioners and training courses are available. The key is to understand the key steps of the procedure and to do those steps appropriately.

The key steps of the laparoscopic TEP repair for better outcomes and fewer complications are as follows: 1) having an orientation for the location of vessels such as inferior epigastric vessel, external iliac vessels, and aberrant obturator artery (corona mortis) even if it is not shown clearly, 2) enough medial and lateral dissection to ensure that the mesh is fully laid out, 3) knowing the point where vulnerable to bleeding, 4) knowing that bleeding from inferior epigastric artery can be easily handled by clipping very proximal site from the external iliac artery, 5) complete parietalization of the spermatic cord and peritonealization of the hernia sac by dividing internal spermatic fascia, and 6) ascertaining that the mesh is not displaced or folded during deflation of the extraperitoneal space.

The limitation of this study is that this is an experience from single surgeon. A well-designed study on the learning curve of trainees under the supervision by the experienced practitioners should be conducted in the future.

In conclusion, the learning curve for laparoscopic TEP repair is not that prolonged if the operating time is used as a parameter of the learning curve. Instead, the learning curve of laparoscopic TEP repair was about 40 cases as using the number of procedures which needed help of the supervisor or needed switch the operator to the supervisor as a parameter.

\section{ACKNOWLEDGEMENTS}

This work was supported in part by the University Research Fund.

\section{REFERENCES}

1) Liem M, Halsema JA, van der Graaf Y, Schrijvers AJ, van Vroonhoven TJ. Cost-effectiveness of extraperitoneal laparoscopic inguinal hernia repair: a randomized comparison with conventional herniorrhaphy. Ann Surg 1997;226:668-676.

2) Heikkinen TJ, Haukipuro K, Koivukangus P, Hulkko A. A prospective randomized outcome and cost comparison of totally extraperitoneal endoscopic hernioplasty versus Lichtenstein hernia operation among employed patients. Surg Laparosc Endosc Percutan Tech 1998;8:338-344.

3) Chung RS, Rowland DY. Meta-analyses of randomized controlled trials of laparoscopic vs conventional inguinal hernia repairs. Surg Endosc 1999;13:689-694.

4) Feliu-Pala X, Martin-Gomez M, Morales-Conde S, FernandezSallent E. The impact of the surgeon's experience on the results of laparoscopic hernia repair . Surg Endosc 2001;15:1467-1470.

5) Voitk AJ. The learning curve in laparoscopic inguinal hernia repair for the community general surgeon. Can J Surg 1998;41:446-450.

6) Edwards CC, Bailey RW. Laparoscopic hernia repair: the learning curve. Surg Laparosc Endosc Percutan Tech 2000;10:149-153.

7) Lau H, Patil NG, Yuen WK, Lee F. Learning curve for unilateral endoscopic totally extraperitoneal (TEP) inguinal hernioplast. Surg Endosc 2002;16:1724-1728.

8) Heidenberg J, Kendrick ML, Meile T, Farley DR. Totally extraperitoneal (TEP) approach for inguinal hernia: the favorable learning curve for trainees. Curr Surg 2003;60:65-68.

9) Choi YY, Kim Z, Hur KY. Learning curve for laparoscopic totally extraperitoneal repair of inguinal hernia. Can J Surg 2012;55:3336.

10) Kim MJ, Hur KY. Laparoscopic totally extraperitoneal inguinal hernia repair: 10-year experience of a single surgeon. Surg Laparosc Endosc Percutan Tech 2013;23:51-54.

11) EU Hernia Trialists Collaboration. Laparoscopic compared with open methods of groin hernia repair: systematic review of randomized controlled trials. Br J Surg 2000; 87:860-867.

12) Liem MSL, van der Graaf Y, van Steensel CJ, et al. Comparision of conventional anterior surgery and laparoscopic surgery for inguinal hernia repair. N Engl J Med 1997;336:1541-1547.

13) Lichtenstein IL, Shulman AG, Amid PK. Laparoscopic hernioplasty. Arch Surg 1991;126:1449.

14) Liem MS, van Steensel CJ, Boelhouwer RU, et al. The learning curve for totally extraperitoneal laparoscopic inguinal hernia repair. Am J Surg 1996;171:281-285.

15) Swanstrom LL. Laparoscopic Herniorrhaphy. Surg Cl N Am 1996;76:483-491.

16) Argote L, Epple D. Learning curves in manufacturing. Science 1990;247:920-924.

17) Moore MJ, Bennett CL. The learning curve for laparoscopic cholecystectomy. The Southern Surgeons Club. Am J Surg 1995;170: 
55-59.

18) Schlachta CM, Mammzza J, Seshadri PA, Cadeddu M, Gregoire R, Poulin EC. Defining a learning curve for laparoscopic colorectal resections. Dis Colon Rectum 2001;44:217-222.

19) Kim MC, Jung GJ, Kim HH. Learning curve of laparoscopy assisted distal gastrectomy with systemic lymphadenectomy for early gastric cancer. World J Gastroenterol 2005;11:7508-7511.

20) Lal P, Kajla RK, Chander J, Ramteke VK. Laparoscopic total extraperitoneal (TEP) inguinal hernia repair: overcoming the learning curve. Surg Endosc 2004;18:642-645. 